\title{
Produtividade de culturas e resistência à penetração de Argissolo Vermelho sob diferentes manejos
}

\author{
Cláudia Liane Rodrigues de Lima(1), Dalvan José Reinert( ${ }^{(2)}$, José Miguel Reichert(2) \\ e Luis Eduardo Akiyoshi Sanches Suzuki ${ }^{(1)}$
}

\begin{abstract}
(1)Universidade Federal de Pelotas, Campus Universitário, s/no, Caixa Postal 354, CEP 96010-900 Pelotas, RS, E-mail: clrlima@yahoo.com.br du_suzuki@hotmail.com (2)Universidade Federal de Santa Maria, Centro de Ciências Rurais, Departamento de Solos, Avenida Roraima, № 1.000, Bairro Camobi, CEP 97105-900 Santa Maria, RS. E-mail: dalvan@smail.ufsm.br, reichert@smail.ufsm.br
\end{abstract}

Resumo - O objetivo deste trabalho foi avaliar a relação entre parâmetros de plantas de soja e feijão e a resistência do solo à penetração de Argissolo Vermelho distrófico arênico sob semeadura direta e convencional. Em experimento implantado em 1989 em semeadura direta, foram aplicadas diferentes passadas de máquina de $10 \mathrm{Mg}$ e realizados diferentes preparos do solo em delineamento inteiramente casualizado. Foram quantificados o índice de velocidade de emergência, o índice de área foliar, a altura e a produtividade de grãos de soja e de feijão e a resistência do solo à penetração. O índice de área foliar, a altura e a produtividade do feijão são influenciados pela resistência do solo à penetração em 46, 51 e $59 \%$, respectivamente, enquanto $55 \%$ da variação da altura da soja é explicada pela resistência à penetração. Indica-se um valor crítico de resistência à penetração de aproximadamente 1,7 e 1,9 MPa no que se refere ao crescimento e à produtividade de grãos de feijão e de soja, respectivamente.

Termos para indexação: Glycine max, Phaseolus vulgaris, plantio convencional, plantio direto.

\section{Yield of crops and soil resistance to penetration of the Alfisol under different management}

\begin{abstract}
The objective of this work was to evaluate the relationship among soybean and bean plant parameters and soil resistance to penetration of an Alfisol under no-tillage and conventional systems. In an experiment deployed under no-tillage in 1989, different wheel tracks of a 10-Mg machine were applied and different soil management systems were used in completely randomized design. Emergence speed index, foliar area index, height and yield of soybean and bean crops and soil resistance to penetration were quantified. The influence of soil resistance to penetration in foliar area index, height and productivity of beans is of 46,51 and $59 \%$ respectively, whereas $55 \%$ of the height variation in soybean is explained by soil resistance to penetration. The critical value of soil resistance to penetration indicated for bean and soybean growth and yield is of approximately 1.7 and $1.9 \mathrm{MPa}$ respectively.
\end{abstract}

Index terms: Glycine max, Phaseolus vulgaris, conventional system, no-tillage.

\section{Introdução}

A qualidade física do solo e o desenvolvimento de plantas estão relacionados à compactação do solo (Strudley et al., 2008). Alterações na estrutura do solo devido à compactação por pressão de máquinas (Lima et al., 2006; Botta et al., 2008) influenciam o fluxo de gases, de água e de calor, a resistência do solo à penetração (RP), o crescimento radicular e de plântulas e a produtividade das culturas (Botta et al., 2006). A RP tem sido um dos atributos físicos frequentemente utilizados na quantificação da qualidade estrutural adequada ao crescimento de plantas (Pringle \& Lark,
2007; Suzuki et al., 2007) em solo sob semeadura direta e convencional (De Vita et al., 2007).

$\mathrm{O}$ efeito de máquinas sobre a compactação tem sido positivamente caracterizado pela RP(Streck et al., 2004). Trabalhos como o de Genro Junior et al. (2004) destacam a importância da RP na identificação de camadas compactadas pelo uso do solo. Incrementos na RP aumentam a energia necessária para o desenvolvimento das raízes das plantas e diminuem a elongação e o crescimento radicular (Lipiec \& Hatano, 2003). No entanto, diversas restrições têm sido apresentadas com relação à indicação de valores críticos de RP para o desenvolvimento de raízes (Sinnett et al., 2008). 
O motivo dessas restrições advém principalmente de a RP ser influenciada pela umidade, densidade e textura do solo (Busscher, 1990; Pabin et al., 1998).

Além de Taylor et al. (1966), que, em condições controladas, demonstraram que a RP superior a $2 \mathrm{MPa}$ é restritiva, Meroto Junior \& Mundstock (1999) também verificaram que a RP de $2 \mathrm{MPa}$ ocasionou redução da massa de matéria seca de raízes, da parte aérea e do comprimento radicular do trigo, enquanto que, para 3,5 $\mathrm{MPa}$, as restrições foram mais severas. Beutler \& Centurion (2004) observaram, em Latossolo, que o diâmetro médio e a massa de matéria seca de raízes da soja aumentaram até um valor de RP de 2,07 e 1,99 MPa, respectivamente, e que a redução da produtividade de grãos de soja ocorreu a $0,85 \mathrm{MPa}$. No entanto, De Maria et al. (1999) relataram que uma RP de 2,09 MPa avaliada em campo poderia determinar redução do crescimento radicular da soja em Latossolo.

O objetivo deste trabalho foi avaliar e associar valores de resistência do solo à penetração, medida em campo, com o crescimento e produtividade das culturas de soja e de feijão em um Argissolo Vermelho distrófico arênico sob semeadura direta e convencional.

\section{Material e Métodos}

O trabalho foi realizado na área experimental do Departamento de Solos da Universidade Federal de Santa Maria, RS $\left(29^{\circ} 41^{\prime} \mathrm{S}\right.$; 53⒋ $48^{\prime} \mathrm{O}$; altitude de $95 \mathrm{~m})$. O clima da região é caracterizado como Cfa (subtropical úmido sem estiagens) de acordo com a classificação de Köppen, a temperatura média do mês mais quente é superior a $22^{\circ} \mathrm{C}$, e as temperaturas do mês mais frio variam entre -3 e $18^{\circ} \mathrm{C}$. O solo foi classificado como Argissolo Vermelho distrófico arênico (Santos et al., 2006) de textura superficial (até $0,30 \mathrm{~m})$ franco-arenosa $\left(81 \mathrm{~g} \mathrm{~kg}^{-1}\right.$ de argila, $291 \mathrm{~g} \mathrm{~kg}^{-1}$ de silte e $628 \mathrm{~g} \mathrm{~kg}^{-1}$ de areia).

$\mathrm{O}$ experimento com a cultura da soja (Glycine $\max$ (L.) Merrill), cultivar Fepagro RS 10 (12 parcelas), e do feijão (Phaseolus vulgaris L.), cultivar Macotaço tipo III (24 parcelas semeadas com espaçamento de $0,45 \mathrm{~m}$ entre linhas), foi distribuído em parcelas de $6 \times 7 \mathrm{~m}$, em delineamento inteiramente casualizado, com quatro repetições. Os tratamentos foram: SD, sistema de semeadura direta desde o ano de 1989; SDE1, SD escarificado em 2002 e em 2004; SDE2, SD escarificado em 2004; SDC1, SD com quatro passadas de máquina de aproximadamente $10 \mathrm{Mg}$ no ano agrícola de 2001/2002; SDC2, SD com quatro passadas de máquina de aproximadamente $10 \mathrm{Mg}$ no ano agrícola de 2002/2003; SDC3, SD com quatro passadas de máquina de aproximadamente $10 \mathrm{Mg}$ nos anos agrícolas de 2001/2002 e 2002/2003. Adicionalmente, parcelas adjacentes ao experimento em delineamento inteiramente casualizado (quatro repetições) foram distribuídas em um sistema convencional de preparo (SC) (uma aração e duas gradagens) de solo com soja e feijão. Nesse sentido, foram testados diferentes manejos, designados por quatro tratamentos em solo com soja (SD, SDE2, SDC3 e SC) e seis com feijão (SD, SDE1, SDE2, SDC1, SDC2 e SC).

A semeadura da soja e do feijão foi realizada em dezembro de 2004. No ano agrícola 2004/2005, foi determinado, para ambas as culturas, o índice de velocidade de emergência (IVEM), em uma contagem de plântulas emergidas no intervalo de dois dias (duas repetições por parcela), utilizando a metodologia de Popinigs (1985). Aos 21, 27, 39, 46 e 56 dias após a semeadura (DAS) da soja e aos 20, 26, 38, 45 e 55 DAS do feijão, foram quantificados (com três repetições por parcela) a área foliar (AF), o índice de área foliar (IAF) e a altura das plantas (AP). A área foliar (AF) foi estimada pelo comprimento e largura de cada folha. Com a finalidade de se estabelecer uma relação entre a AF real e a observada em campo, foram coletados 30 trifólios no início do desenvolvimento das culturas. A AF real foi determinada com o auxílio de uma mesa digitalizadora acoplada a um microcomputador e do software CR-Siter 2.5. Estimou-se a equação de regressão linear entre a $\mathrm{AF}$ observada e a real para soja $\left(y=0,726 x+0,029, R^{2}=0,99\right)$ e para o feijão $\left(y=0,561 x+0,095, R^{2}=0,98\right)$, obtendo-se o IAF pela relação entre a AF fotossinteticamente ativa e a área superficial do solo ocupada pela planta. AAP foi medida pela distância entre a superfície do solo e o último nó da haste principal da planta. No florescimento das culturas, fase considerada relevante para a definição do potencial produtivo, avaliou-se a resistência à penetração (RP) utilizando um penetrômetro digital CP 20 Ultrasonic Cone Penetrometer, (Rimik, Toowoomba, Austrália) com ponta cônica e ângulo de penetração de $30^{\circ} \mathrm{e}$ armazenamento eletrônico dos dados a cada $0,015 \mathrm{~m}$ até a profundidade de $0,40 \mathrm{~m}$. A determinação foi efetuada em cada parcela e a cada $0,10,0,20$ e $0,30 \mathrm{~m}$ para a esquerda e para a direita da linha de semeadura. 
Os dados foram apresentados na forma de mapas de contorno em duas dimensões, elaborados a partir da interpolação de resultados por krigagem e com auxílio do Surfer 8.0 (Golden Software, 2002). A umidade volumétrica não diferiu entre os tratamentos, obtendose valores médios de 0,$20 ; 0,21 ; 0,22$ e $0,23 \mathrm{~m}^{3} \mathrm{~m}^{-3}$ para as camadas de 0,00 a 0,$05 ; 0,10$ a 0,$15 ; 0,20$ a 0,25 e 0,30 a $0,35 \mathrm{~m}$, respectivamente. Esses valores de umidade volumétrica estão próximos da capacidade de campo $\left(0,23 \mathrm{~m}^{3} \mathrm{~m}^{-3}\right)$ avaliada neste experimento.

Para a quantificação da produtividade, coletaram-se três linhas de $2 \mathrm{~m}$ de comprimento de cada cultura em cada parcela, e os valores de umidade dos grãos foram corrigidos para $13 \%$ (base úmida).

Os dados foram avaliados a partir de contrastes ortogonais e de regressões não lineares a $5 \%$ de probabilidade. Os contrastes ortogonais para o IAF e AP (Tabela 1) foram efetuados para o último período de avaliação (56 e 55 DAS da soja e feijão, respectivamente). As análises de regressão foram efetuadas para avaliar a relação entre a RP quantificada na linha da cultura e os atributos das plantas (IVEM, IAF, AP e produtividade).

\section{Resultados e Discussão}

Com base nos contrastes ortogonais, houve diferença entre os valores médios de índice de velocidade de emergência (IVEM) da soja (CV = 36,48\%) entre SD, SDC3 e SC (Tabela 1). Menores valores de IVEM foram proporcionados pelo sistema convencional (SC) e diferiram de SD e SDE2 (Figura 1 A). O maior contato solo-semente na semeadura da cultura, proporcionado pelo SD, possivelmente contribuiu para o maior IVEM. O contato solo-semente associado à adequada macroporosidade e à agregação é relevante para uma adequada emergência e estabelecimento das culturas (Brown et al., 1996). Por outro lado, o revolvimento do solo e a ausência de cobertura vegetal proporcionaram selamento e encrostamento superficial, ocasionando diminuição no IVEM no SC. Considerando os valores médios de IVEM da cultura do feijão $(C V=27,35 \%)$, observou-se que o maior IVEM, obtido no SDC1 (Figura $1 \mathrm{~B}$ ), contrastou significativamente com SD, SC, SDE2 e SDC2 (Tabela 1). A escarificação e o preparo convencional proporcionaram condições menos adequadas para a emergência, indicadas pelos valores de IVEM do feijão.

Tabela 1. Valor de F e significância dos contrastes entre diferentes manejos para índice de velocidade de emergência (IVEM), índice de área foliar (IAF), altura de planta (AP) e produtividade de soja e de feijão.

\begin{tabular}{|c|c|c|c|c|}
\hline Contrastes $^{(1)}$ & IVEM & IAF & AP & Produtividade \\
\hline \multicolumn{5}{|c|}{ Soja } \\
\hline SD x SDE2 & $2,55^{\mathrm{ns}}$ & $2,33^{\mathrm{ns}}$ & $0,41^{\mathrm{ns}}$ & $0,71^{\mathrm{ns}}$ \\
\hline $\mathrm{SD} \times \mathrm{SDC} 3$ & $9,63 * *$ & $3,90^{\mathrm{ns}}$ & $1,21^{\mathrm{ns}}$ & $1,60^{\mathrm{ns}}$ \\
\hline $\mathrm{SD} \times \mathrm{SC}$ & $16,76^{* *}$ & $0,61^{\mathrm{ns}}$ & $0,11^{\mathrm{ns}}$ & $3,73^{\mathrm{ns}}$ \\
\hline SDE2 $x$ SDC 3 & $2,28^{\mathrm{ns}}$ & $0,20^{\mathrm{ns}}$ & $0,22^{\text {ns }}$ & $0,18^{\text {ns }}$ \\
\hline SDE2 $\times$ SC & $7,79^{*}$ & $0,40^{\text {ns }}$ & $0,85^{\text {ns }}$ & $1,18^{\text {ns }}$ \\
\hline SDC $3 \times \mathrm{SC}$ & $2,43^{\text {ns }}$ & $1,10^{\mathrm{ns}}$ & $1,82^{\text {ns }}$ & $0,44^{\mathrm{ns}}$ \\
\hline \multicolumn{5}{|c|}{ Feijão } \\
\hline SD x SDE2 & $5,80^{*}$ & $1,20^{\mathrm{ns}}$ & $0,01^{\mathrm{ns}}$ & $4,15^{\text {ns }}$ \\
\hline SD x SDC1 & $4,76^{*}$ & $0,11^{\mathrm{ns}}$ & $0,03^{\mathrm{ns}}$ & $2,06^{\mathrm{ns}}$ \\
\hline SD x SDE1 & $0,07^{\mathrm{ns}}$ & $0,52^{\text {ns }}$ & $2,54^{\mathrm{ns}}$ & $0,36^{\mathrm{ns}}$ \\
\hline $\mathrm{SD} \times \mathrm{SDC} 2$ & $0,05^{\text {ns }}$ & $0,17^{\mathrm{ns}}$ & $0,63^{\text {ns }}$ & $0,00^{\text {ns }}$ \\
\hline $\mathrm{SD} \times \mathrm{SC}$ & $1,83^{\mathrm{ns}}$ & $11,62 * *$ & $7,33^{\text {ns }}$ & $0,33^{\mathrm{ns}}$ \\
\hline SDE2 $\times$ SDC1 & $15,79 * *$ & $0,63^{\mathrm{ns}}$ & $0,04^{\mathrm{ns}}$ & $9,03 * *$ \\
\hline SDE2 $\times$ SDE1 & $5,34^{*}$ & $0,25^{\mathrm{ns}}$ & $1,62^{\mathrm{ns}}$ & $5,20 *$ \\
\hline SDE2 $\times$ SDC2 & $3,59^{\mathrm{ns}}$ & $0,31^{\mathrm{ns}}$ & $0,52^{\text {ns }}$ & $2,20^{\mathrm{ns}}$ \\
\hline SDE2 $\times$ SC & $0,22^{\text {ns }}$ & $7,32 *$ & $6,68^{*}$ & $1,61^{\mathrm{ns}}$ \\
\hline SDC1 1 SDE1 & $2,77^{\mathrm{ns}}$ & $0,13^{\mathrm{ns}}$ & $1,70^{\mathrm{ns}}$ & $0,53^{\text {ns }}$ \\
\hline SDC1 1 SDC2 & $4,32 *$ & $0,02^{\text {ns }}$ & $0,39^{\text {ns }}$ & $0,95^{\mathrm{ns}}$ \\
\hline $\mathrm{SDC} 1 \times \mathrm{SC}$ & $7,71 * *$ & $8,83^{*}$ & $7,88^{*}$ & $3,01^{\mathrm{ns}}$ \\
\hline SDE1 x SDC2 & $0,17^{\mathrm{ns}}$ & $0,02^{\text {ns }}$ & $0,19^{\text {ns }}$ & $0,14^{\mathrm{ns}}$ \\
\hline SDE1 x SC & $2,01^{\mathrm{ns}}$ & $7,16^{*}$ & $1,34^{\mathrm{ns}}$ & $1,02^{\text {ns }}$ \\
\hline SDC $2 \times S C$ & $1,17^{\text {ns }}$ & $5,99 *$ & $1,92^{\text {ns }}$ & $0,20^{\text {ns }}$ \\
\hline
\end{tabular}

${ }^{(1)} \mathrm{SD}$, sistema de semeadura direta desde o ano de 1989; SDE1, SD escarificado em 2002 e 2004; SDE2, SD escarificado em dezembro de 2004; SDC1, SDC2 e SDC3, SDs com quatro passadas de máquina de aproximadamente $10 \mathrm{Mg}$ nos anos agrícolas de 2001/2002, 2002/2003, 2001/2002 e 2002/2003, respectivamente; SC, sistema de preparo do solo convencional. ${ }^{n}$ Não significativo. * $\mathrm{e}^{* *}$ Significativo a 5 e $1 \%$ de probabilidade, respectivamente. 
$\mathrm{O}$ índice de área foliar, a altura de planta e a produtividade de grãos de soja não foram influenciados pelo manejo de solo (Tabela 1). Secco et al. (2004) também concluíram que a produtividade de grãos de soja em um Latossolo Vermelho sob semeadura direta não foi afetada pela compactação do solo. Flores et al. (2007), similarmente, observaram que não houve influência do sistema de manejo no estabelecimento e na produtividade de grãos de soja. $\mathrm{O}$ valor médio superior de IAF do feijão no SC observado no último período de avaliação (Figura $1 \mathrm{D}$ ) contrastou com SD, SDE1, SDE2, SDC1 e SDC2 (Tabela 1), o que evidencia que o revolvimento do solo (SC) favorece maior área foliar em comparação aos demais manejos. O SC apresentou valores de altura de plantas (AP) mais elevados para o feijão no último período de avaliação
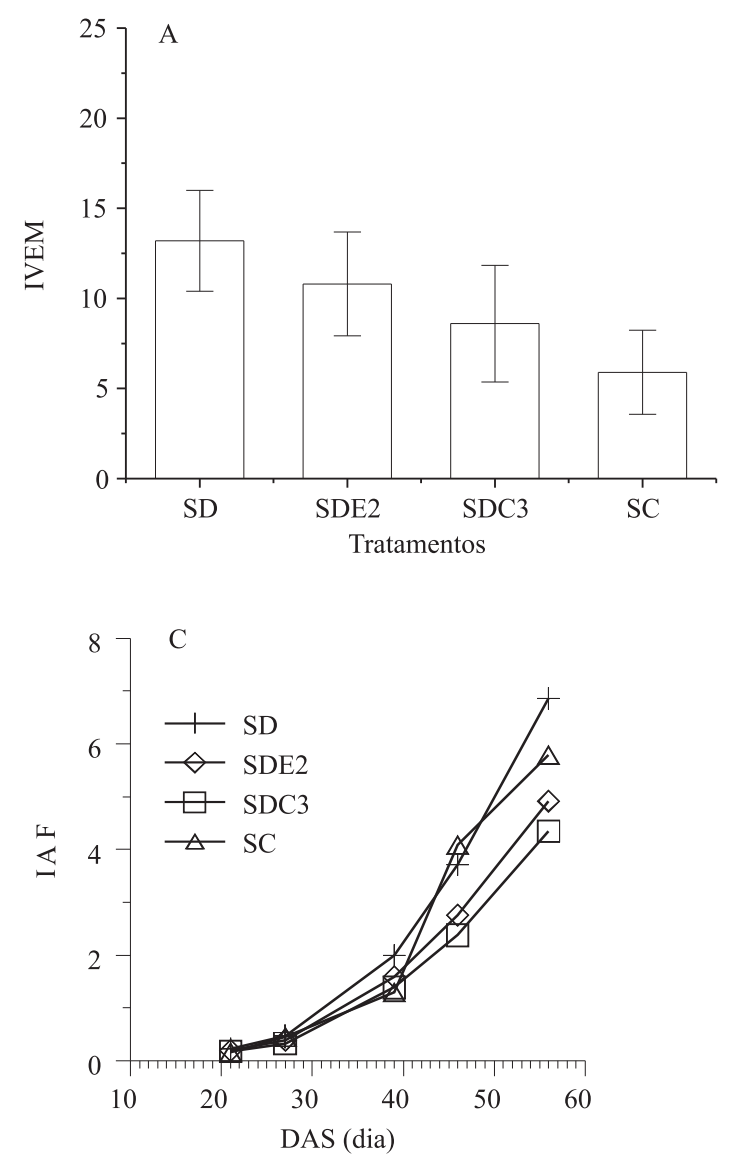

(Figura 2 B) em relação a SDE2 e SDC1 (Tabela 1). A produtividade dessa cultura (Figura $2 \mathrm{D}$ ) ainda apresentou diferenças significativas para os contrastes entre SDE2 versus SDE1 e SDC1 (Tabela 1).

Para melhor entendimento da dependência de atributos relacionados à planta e solo, estabeleceramse regressões entre o IAF, a AP e a produtividade das culturas com a resistência do solo à penetração (RP) (Figura 3). Todas as relações foram polinomiais de segundo grau, o que corrobora observações de Beutler $\&$ Centurion (2004).

Somente a altura da soja foi significativamente influenciada pela RP. A partir do cálculo da primeira derivada dessa equação ajustada, observou-se que valores de RP de aproximadamente 1,3 $\mathrm{MPa}$ proporcionaram altura máxima $(\mathrm{AP}=0,47 \mathrm{~m})$
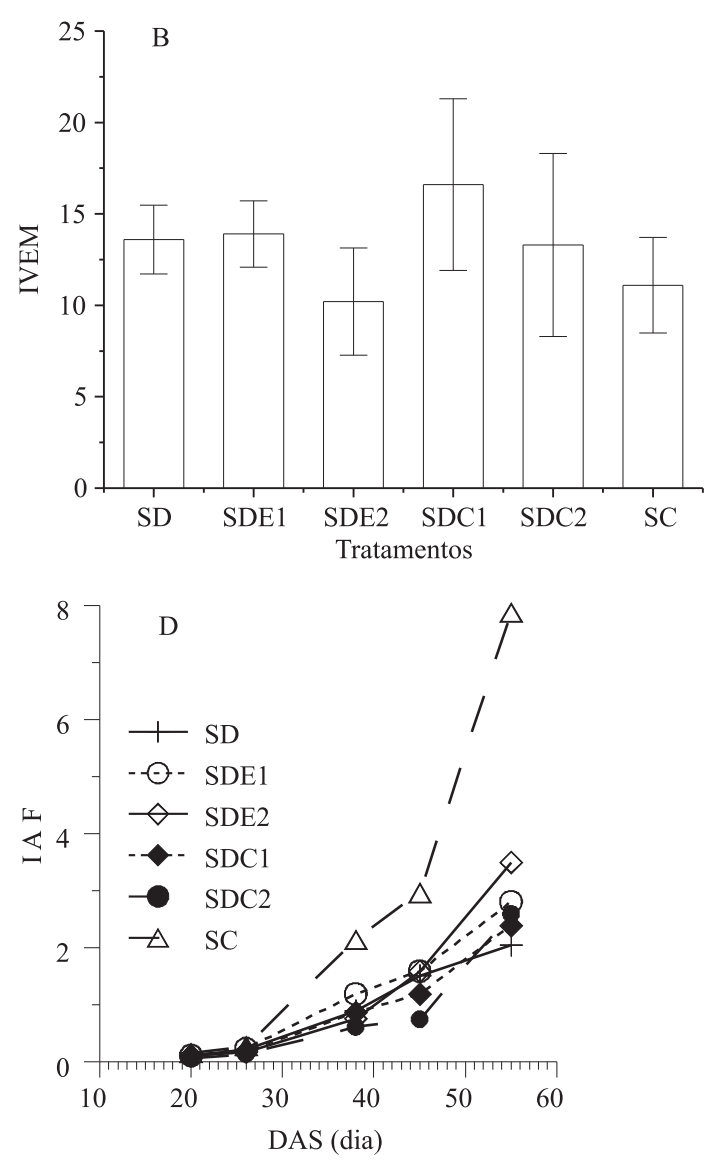

Figura 1. Índice de velocidade de emergência (IVEM) da (A) soja e do (B) feijão e índice de área foliar (IAF) aos 21, 27, 39, 46 e 56 dias após a semeadura (DAS) da (C) soja e aos 20, 26, 38, 45 e 55 DAS do (D) feijão em um Argissolo Vermelho sob diferentes manejos. SD, sistema de semeadura direta desde o ano de 1989; SDE1, SD escarificado em 2002 e 2004; SDE2, SD escarificado em dezembro de 2004; SDC1, SDC2 e SDC3, SDs com quatro passadas de máquina de aproximadamente $10 \mathrm{Mg}$ nos anos agrícolas de 2001/2002, 2002/2003, 2001/2002 e 2002/2003, respectivamente; SC, sistema de preparo do solo convencional. Barras verticais indicam o desvio padrão. 
(Figura 3 C). No cultivo do feijão, verificou-se que o IAF, a AP e a produtividade de grãos dependeram da RP (Figuras 3 B, D e F) e apresentaram valores médios máximos entre 1,2 e 1,3 $\mathrm{MPa}$.

Apesar de ainda existirem dúvidas sobre valores de resistência do solo à penetração considerados críticos ao desenvolvimento das culturas (Earl, 1997), resultados semelhantes foram obtidos por Grant \& Lanfond (1993), os quais sugerem um intervalo de RP com valores superiores a 1,5 e 3,0 MPa como impeditivo ao crescimento radicular. Carvalho et al. (2006) relatam que valores de RP variando entre 1,3 e 2,9 MPa não restringiram a produtividade de grãos da cultura do feijão em um Latossolo. Similarmente, Rosolem et al. (1999) concluíram que a RP de 1,3 MPa em Latossolo reduziu o crescimento das raízes
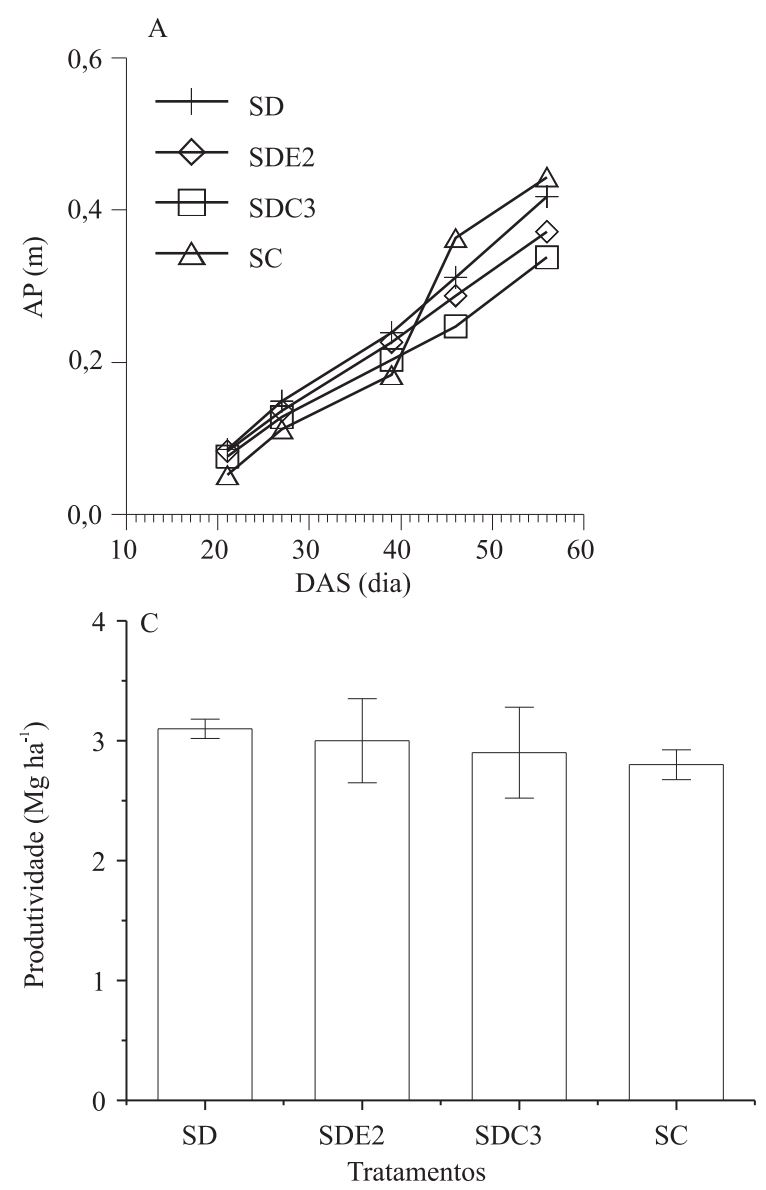

seminais adventícias do milho pela metade, o que corrobora os resultados obtidos por Dexter (1987). Outros estudos relatam que o desenvolvimento de raízes é impedido em situações de valores que excedem 1,3 e 1,5 MPa (Zou et al., 2001).

Considerando-se as condições deste trabalho de forma geral, e para uma RP crítica (RPc) ao desenvolvimento de soja e de feijão de aproximadamente $1,3 \mathrm{MPa}$, foi identificada no solo com SD da soja uma grande área vertical do perfil de solo com valores superiores aos da RPc (Figura 4 A). O SDE2 proporcionou zonas com resistência superior à $\mathrm{RPc}$ a uma profundidade de $0,20 \mathrm{~m}$ (Figura $4 \mathrm{~B}$ ), similarmente a SDC3 e SC (Figura 4 C e D).

Em áreas de cultivo do feijão, a camada de maior $\mathrm{RP}$ no SD esteve próxima a $0,15 \mathrm{~m}$ (Figura $5 \mathrm{~A}$ ).
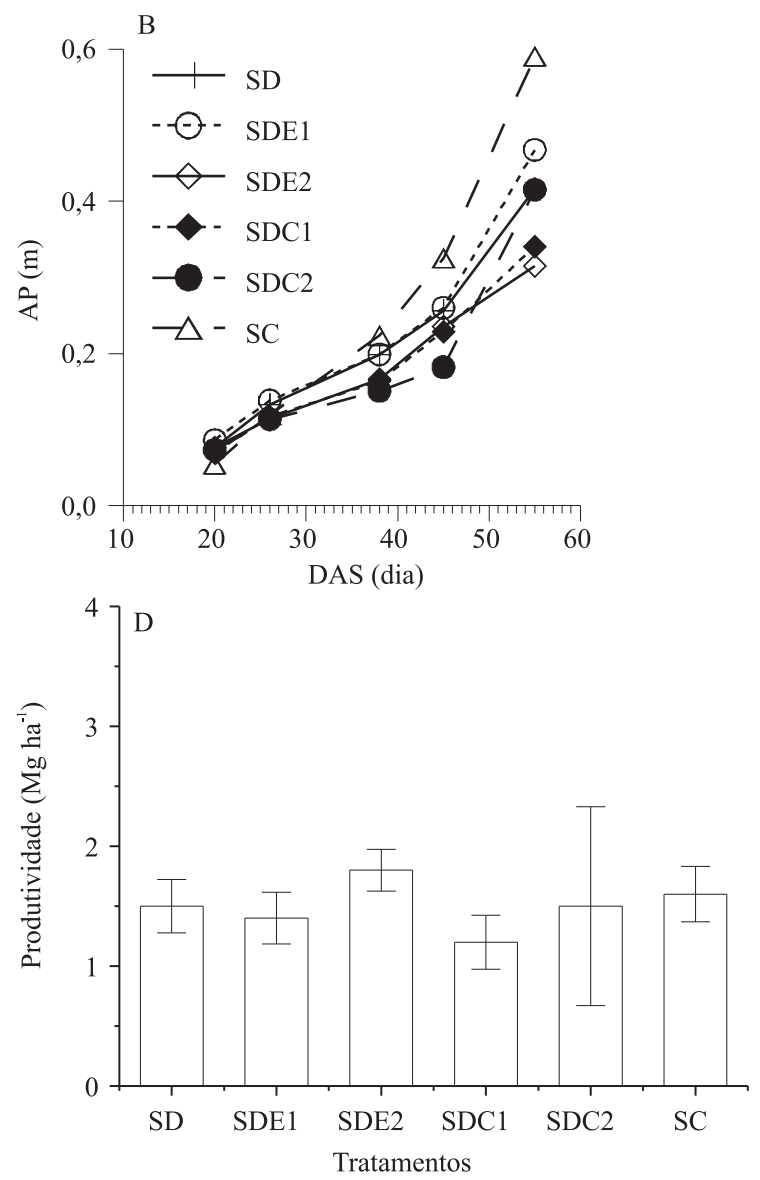

Figura 2. Altura de plantas (AP) aos 21, 27, 39, 46 e 56 dias após a semeadura (DAS) da (A) soja e aos 20, $26,38,45$ e 55 DAS do (B) feijão e produtividade de grãos de (C) soja e (D) feijão em um Argissolo Vermelho sob diferentes manejos. SD, sistema de semeadura direta desde o ano de 1989; SDE1, SD escarificado em 2002 e 2004; SDE2, SD escarificado em dezembro de 2004; SDC1, SDC2 e SDC3, SDs com quatro passadas de máquina de aproximadamente $10 \mathrm{Mg}$ nos anos agícolas de 2001/2002, 2002/2003, 2001/2002, e 2002/2003, respectivamente; SC, sistema de preparo do solo convencional. Barras verticais indicam o desvio padrão. 
No SDE2, surgiram várias áreas de RPc (Figura 5 B); ocorreram valores de RPc a $0,10 \mathrm{~m}$ no $\mathrm{SDC}$ e a $0,15 \mathrm{~m}$ no SDE1 (Figura $5 \mathrm{C}$ e D); no SDC2, a camada de resistência superior a 1,5 MPa iniciou-se a $0,08 \mathrm{~m}$ (Figura $5 \mathrm{E}$ ); e, no SC, o início foi próximo a $0,25 \mathrm{~m}$ (Figura $5 \mathrm{~F}$ ).
Por outro lado, se for considerada aceitável uma redução de produtividade de $10 \%$, ou seja, uma produtividade mínima de $90 \%$ daquela considerada máxima, conforme critérios utilizados em trabalhos de adubação de solo, a produtividade mínima aceitável de feijão seria de 1,51 $\mathrm{Mg} \mathrm{ha}^{-1}$ (Figura $3 \mathrm{~F}$ ).
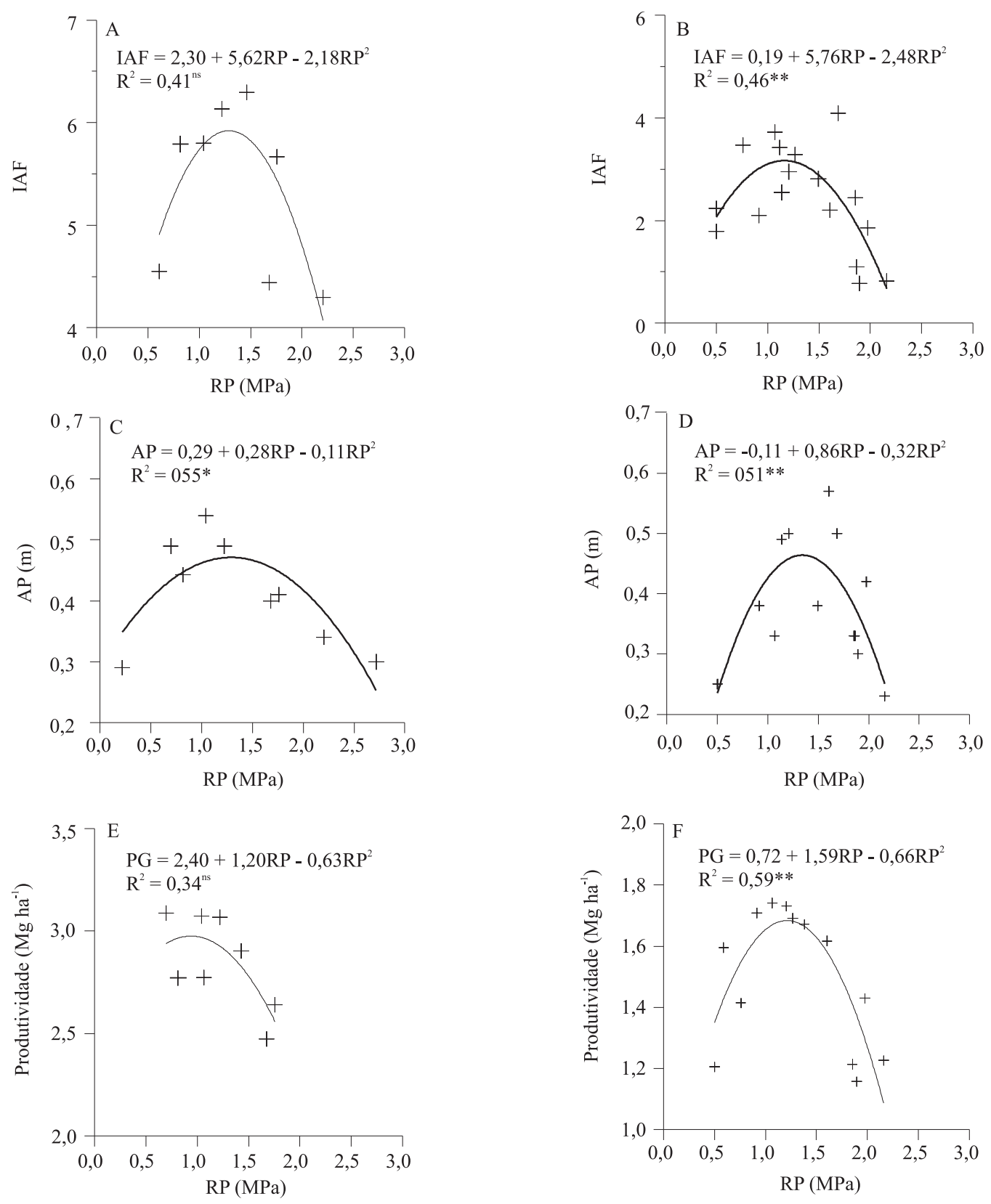

Figura 3. Relação entre resistência do solo à penetração (RP) de Argissolo Vermelho e índice de área foliar (IAF) de (A) soja e (B) feijão, altura (AP) de (C) soja e (D) feijão e produtividade

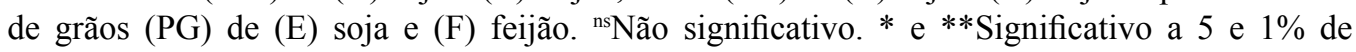
probabilidade, respectivamente. 
Essa produtividade ocorreria entre 0,7 e 1,7 MPa. Nesse intervalo seriam obtidas produtividades acima de $90 \%$ da máxima, e uma RP de 1,7 MPa seria crítica para a cultura do feijão. Similarmente, $90 \%$ da AP máxima da soja (Figura 3 C) $(0,42 \mathrm{~m})$ apresentaria limites de RP entre 0,6 e 1,9 MPa.

Com base nesse critério, valores de RP de 1,7 e 1,9 MPa podem ser considerados limitantes para o desenvolvimento das plantas de feijão e da soja, respectivamente. Sendo assim, para a cultura da soja, maiores restrições foram observadas nos sistemas SDE2, SDC3 e SC (Figuras 4 B, C e D), e todos os sistemas de manejo demonstraram certa restrição para o desenvolvimento da cultura do feijão (Figura 5).

Assim, verifica-se que o solo poderá apresentar camadas com resistências variáveis, o que possibilita o crescimento de raízes (Queiroz-Voltan et al., 2000). Menores valores de resistência à penetração podem estar associados à maior porosidade de solo (Boone et al., 1994), o que proporciona um desenvolvimento adequado de raízes.

A
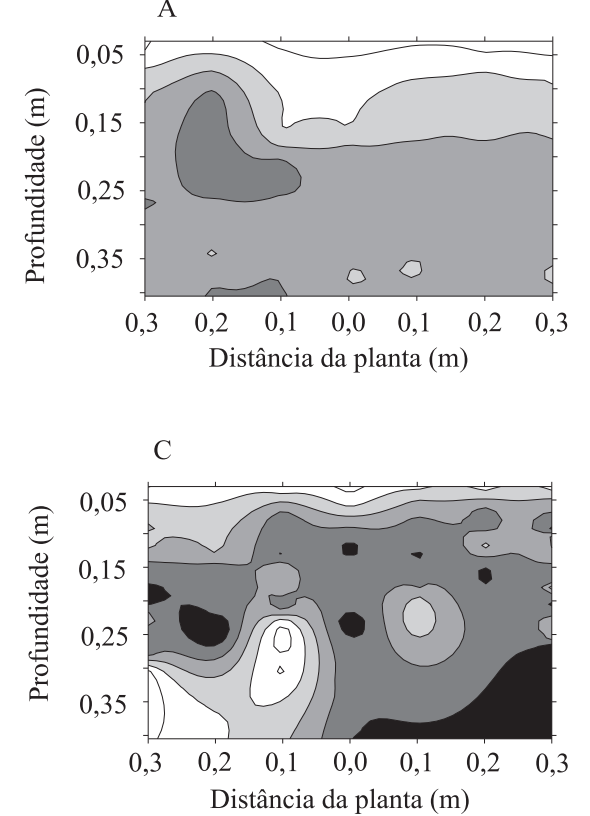

Práticas que previnem a compactação são sugeridas por Hamza \& Anderson (2005), como a rotação de culturas, a diminuição da carga aplicada por máquinas agrícolas, a restrição no confinamento do tráfego e o aumento da área de contato do rodado sobre o solo.

O presente trabalho contribui para o entendimento da influência da RP no crescimento aéreo e radicular e na produtividade de culturas. Um monitoramento da resistência à penetração (Tavares Filho \& Ribon, 2008), associado ao valor de umidade do solo (Souza et al., 2006) durante todo o ciclo da cultura, pode ser ideal para o estabelecimento de um adequado desenvolvimento de plantas. Avaliações de médio e longo prazo podem indicar mudanças dinâmicas da qualidade física do solo, ocasionadas pelos sistemas de preparo e de manejo do solo e da cultura.

Considera-se que há necessidade de estudos adicionais para o conhecimento das alterações da RP impostas por sistemas de manejo (Amuri \& Brye, 2008) e do impacto dessas alterações no desenvolvimento e na produtividade de diferentes culturas.
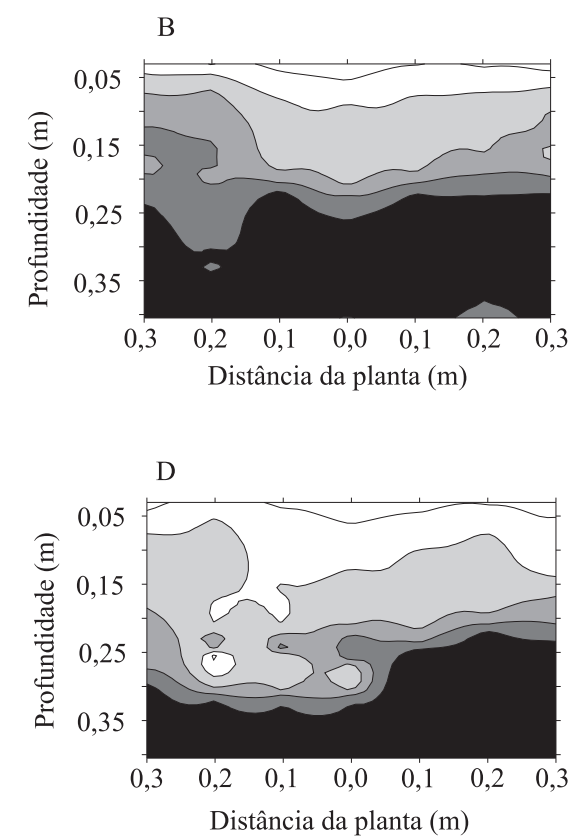

$\begin{array}{lllll}0,5 & 1,0 & 1,5 & 2,0 & 2,5\end{array}$

$\mathrm{RP}(\mathrm{MPa})$

Figura 4. Resistência do solo à penetração em um perfil $(0,35 \mathrm{~m})$ de Argissolo Vermelho sob soja nos manejos: (A) SD, semeadura direta desde o ano de 1989; (B) SDE2, SD escarificado em dezembro de 2004; (C) SDC3, SD com quatro passadas de máquina de aproximadamente $10 \mathrm{Mg}$ no ano agrícola 2001/2002 e 2002/2003 e (D) SC, sistema de preparo do solo convencional. 

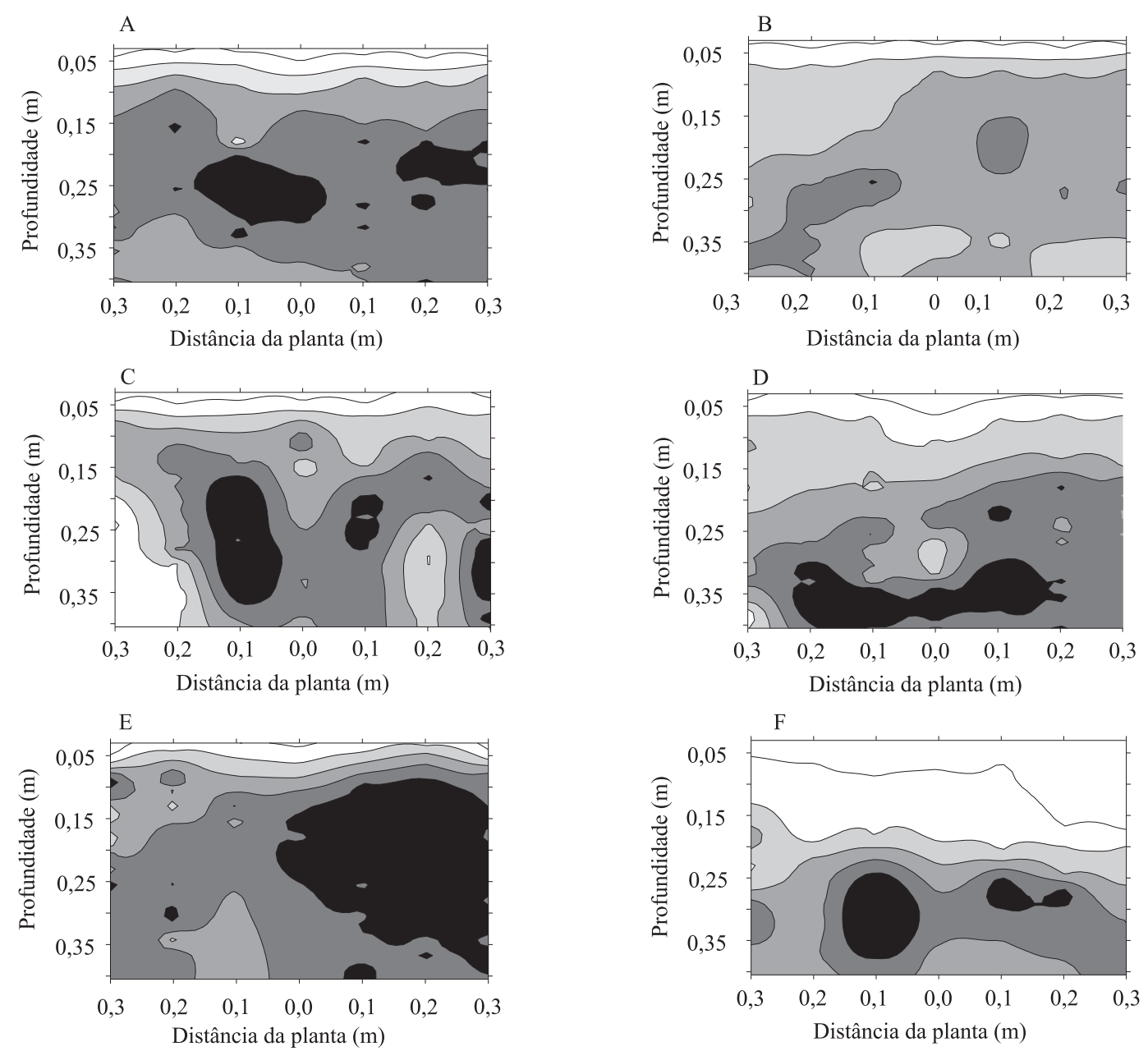

$$
\begin{array}{|cccc|}
\hline 0,5 & 1,0 \quad 1,5 \quad 2,0 & 2,5 \\
& \operatorname{RP}(\mathrm{MPa})
\end{array}
$$

Figura 5. Resistência do solo à penetração em um perfil $(0,35 \mathrm{~m})$ de Argissolo Vermelho sob feijão nos manejos: (A) SD, semeadura direta desde o ano de 1989; (B) SDE2, SD escarificado em dezembro de 2004 e (C) SDC1, SD com quatro passadas de máquina de aproximadamente $10 \mathrm{Mg}$ no ano agrícola 2001/2002; (D) SDE1, SD escarificado em 2002 e 2004; (E) SDC2, SD com quatro passadas de máquina de aproximadamente $10 \mathrm{Mg}$ no ano agrícola 2002/2003 e (F) SC, sistema de preparo do solo convencional.

\section{Conclusões}

1. Nas condições de umidade de solo avaliadas em campo, a resistência do solo à penetração crítica para a produtividade de grãos de feijão é $1,7 \mathrm{MPa}$, e a altura de plantas da soja é influenciada a partir de 1,9 MPa.

2. O índice de velocidade de emergência das plantas de soja e de feijão é menor no sistema convencional de preparo do solo do que no sistema de semeadura direta.

3. O sistema convencional de preparo do solo possibilita maiores valores de índice de área foliar e de altura do feijão em relação ao sistema de semeadura direta.

4. A resistência do solo à penetração influenciou o índice de área foliar, a altura e a produtividade do feijão em 46,51 e $59 \%$, respectivamente. 


\section{Referências}

AMURI, N.; BRYE, K.R. Residue management practice effects on soil penetration resistance in a wheat-soybean double-crop production system. Soil Science, v.173, p.779-791, 2008.

BEUTLER, A.N.; CENTURION, J.F. Compactação do solo no desenvolvimento radicular e na produtividade da soja. Pesquisa Agropecuária Brasileira, v.39, p.581-588, 2004.

BOONE, F.R.; VERMEULEN, G.D.; KROESBERGEN, B. The effect of mechanical impedance and soil aeration as affected by surface loading on the growth of peas. Soil and Tillage Research, v.32, p.237-251, 1994.

BOTTA, G.F.; JORAJURIA, D.; ROSATTO, H.; FERRERO, C. Light tractor traffic frequency on soil compaction in the Rolling Pampa region of Argentina. Soil and Tillage Research, v.86, p.9-14, 2006.

BOTTA, G.F.; RIVERO, D.; TOURN, M.; BELLORA MELCON, F.; POZZOLO, O.; NARDON, G.; BALBUENA, R.; TOLON BECERRA, A.; ROSSATTO, H.; STADLER, S. Soil compaction produced by tractor with radial and cross-ply tyres in two tillage regimes. Soil and Tillage Research, v.101, p.44-51, 2008.

BROWN, A.D.; DEXTER, A.R.; CHAMEN, W.C.T.; SPOOR, G. Effect of soil macroporosity and aggregate size on seed-soil contact. Soil and Tillage Research, v.38, p.203-216, 1996.

BUSSCHER, W.J. Adjustment of flat-tipped penetrometer resistance data to a common water content. Transactions of the ASAE, v.33, p.519-524, 1990.

CARVAlHO, G.J.; CARVAlHO, M. de P.; FREDDI, O. da S.; MARTINS, M.V. Correlação da produtividade do feijão com a resistência à penetração do solo sob plantio direto. Revista Brasileira de Engenharia Agrícola e Ambiental, v.10, p.765-771, 2006.

DE MARIA, I.C.; CASTRO, O.M.; DIAS, H.S. Atributos físicos do solo e crescimento radicular de soja em Latossolo Roxo sob diferentes métodos de preparo do solo. Revista Brasileira de Ciência do Solo, v.23, p.703-709, 1999.

DE VITA, P.; DI PAOLO, E.; FECONDO, G.; DI FONZO, N.; PISANTE, M. No-tillage and conventional tillage effects on durum wheat yield, grain quality and soil moisture content in southern Italy. Soil and Tillage Research, v.92, p.69-78, 2007.

DEXTER, A.R. Mechanics of root growth. Plant and Soil, v.98, p.303-312, 1987.

EARL, R. Prediction of trafficability and workability from soil moisture deficit. Soil and Tillage Research, v.40, p.155-168, 1997.

FLORES, J.P.C.; ANGHINONI, I.; CASSOL, L.C.; CARVALHO, P.C. de F.; LEITE, J.G. Dal B.; FRAGA, T.I. Atributos físicos do solo e rendimento de soja em sistema plantio direto em integração lavoura pecuária com diferentes pressões de pastejo. Revista Brasileira de Ciência do Solo, v.31, p.771-780, 2007.

GENRO JUNIOR, S.A.; REINERT, D.J.; REICHERT, J.M. Variabilidade temporal da resistência à penetração de um Latossolo argiloso sob semeadura direta com rotação de culturas. Revista Brasileira de Ciência do Solo, v.28, p.477-484, 2004.
GOLDEN SOFTWARE. Surfer: countouring and 3D surface mapping for scientists and engineers. Version 8. New York: Golden Software, 2002. 640p.

GRANT, C.A.; LANFOND, G.O. The effects of tillage systems and crop sequences on soil bulk density and penetration resistance on a clay soil in Southern Saskatchewan. Canadian Journal of Soil Science, v.73, p.223-232, 1993.

HAMZA, M.A.; ANDERSON, W.K. Soil compaction in cropping systems: a review of the nature, causes and possible solutions. Soil and Tillage Research, v.82, p.121-145, 2005.

LIMA, C.L.R. de; REINERT, D.J.; REICHERT, J.M.; SUZUKI, L.E.A.S. Compressibilidade de um Argissolo sob plantio direto escarificado e compactado. Ciência Rural, v.36, p.1765-1772, 2006.

LIPIEC, J.; HATANO, R. Quantification of compaction effects on soil physical properties and crop growth. Geoderma, v.116, p.107-136, 2003.

MEROTO JUNIOR, A.; MUNDSTOCK, C.M. Wheat root growth as affected by soil strength. Revista Brasileira de Ciência do Solo, v.23, p.197-202, 1999.

PABIN, J.; LIPIEC, J.; WLODEK, S.; BISKUPSKI, A.; KAUS, A. Critical soil bulk density and strength for pea seedling root growth as related to other soil factors. Soil and Tillage Research, v.46, p.203-208, 1998.

POPINIGIS, F. Fisiologia da semente. 2.ed. Brasília: Abrates, 1985. 289p.

PRINGLE, M.J.; LARK, R.M. Scale- and location-dependent correlations of soil strength and the yield of wheat. Soil and Tillage Research, v.95, p.47-60, 2007.

QUEIROZ-VOLTAN, R.B.; NOGUEIRA, S. dos S.S.; MIRANDA, M.A.C. de. Aspectos da estrutura da raiz e do desenvolvimento de plantas de soja em solos compactados. Pesquisa Agropecuária Brasileira, v.35, p.929-938, 2000.

ROSOLEM, C.A.; FERNANDEZ, E.M.; ANDREOTTI, M.; CRUSCIOL, C.A.C. Crescimento radicular de plântulas de milho afetado pela resistência do solo à penetração. Pesquisa Agropecuária Brasileira, v.34, p.821-828, 1999.

SANTOS, H.G. dos; JACOMINE, P.K.T.; ANJOS, L.H.C. dos; OLIVEIRA, V.A. de; OLIVEIRA, J.B. de; COELHO, M.R.; LUMBRERAS, J.F.; CUNHA, T.J.F. (Ed.). Sistema brasileiro de classificação de solos. 2.ed. Rio de Janeiro: Embrapa Solos, 2006. $306 \mathrm{p}$.

SECCO, D.; REINERT, D.J.; REICHERT, J.M.; ROS, C.O. da. Produtividade de soja e propriedades físicas de um Latossolo submetido a sistemas de manejo e compactação. Revista Brasileira de Ciência do Solo, v.28, p.797-804, 2004.

SINNETT, D.; MORGAN, G.; WILLIAMS, M.; HUTCHINGS, T.R. Soil penetration resistance and tree root development. Soil Use and Management, v.24, p.273-280, 2008.

SOUZA, Z.M. de; CAMPOS, M.C.C.; CAVALCANTE, I.H.L.; MARQUES JÚNIOR, J.; CESARIN, L.G.; SOUZA, S.R. de. Dependência espacial da resistência do solo à penetração e do teor 
de água do solo sob cultivo contínuo de cana-de-açúcar. Ciência Rural, v.36, p.128-134, 2006.

STRECK, C.A.; REINERT, D.J.; REICHERT, J.M.; KAISER, D.R. Modificações em propriedades físicas com a compactação do solo causada pelo tráfego induzido de um trator em plantio direto. Ciência Rural, v.34, p.755-760, 2004.

STRUDLEY, M.W.; GREEN, T.R.; ASCOUGH, J.C. Tillage effects on soil hydraulic properties in space and time: state of the science. Soil and Tillage Research, v.99, p.4-48, 2008.

SUZUKI, L.E.A.S.; REICHERT, J.M.; REINERT, D.J.; LIMA, C.L.R. de. Grau de compactação, propriedades físicas e rendimento de culturas em Latossolo e Argissolo. Pesquisa Agropecuária Brasileira, v.42, p.1159-1167, 2007.

TAVARESFILHO, J.; RIBON,A.A. Resistência do soloà penetração em resposta ao número de amostras e tipo de amostragem. Revista Brasileira de Ciência do Solo, v.32, p.487-494, 2008.

TAYLOR, H.M.; ROBERSON, G.M.; PARKER JUNIOR, J.J. Soil strength-root penetration relations for medium- to coarse-textured soil materials. Soil Science, v.102, p.18-22, 1966.

ZOU, C.; PENFOLD, C.; SANDS, R.; MISRA, R.K.; HUDSON, I. Effects of soil air-filled porosity, soil matric potential and soil strength on primary root growth of radiata pine seedlings. Plant and Soil, v.236, p.105-115, 2001.

$\overline{\text { Recebido em } 17 \text { de fevereiro de } 2009 \text { e aprovado em } 30 \text { de novembro de } 2009}$ 